\title{
Nuclear Magnetic Resonance Spectroscopy as a Dynamical Structural Probe of Hydrogen under High Pressure
}

\author{
Bartomeu Monserrat, ${ }^{1, *}$ Sharon E. Ashbrook, ${ }^{2}$ and Chris J. Pickard ${ }^{3,4}$ \\ ${ }^{1}$ TCM Group, Cavendish Laboratory, University of Cambridge, J. J. Thomson Avenue, Cambridge CB3 OHE, United Kingdom \\ ${ }^{2}$ School of Chemistry, EaStCHEM and Centre of Magnetic Resonance, University of St. Andrews, \\ St. Andrews KY16 9ST, United Kingdom \\ ${ }^{3}$ Department of Materials Science and Metallurgy, University of Cambridge, \\ 27 Charles Babbage Road, Cambridge CB3 OFS, United Kingdom \\ ${ }^{4}$ Advanced Institute for Materials Research, Tohoku University 2-1-1 Katahira, Aoba, Sendai, 980-8577, Japan
}

(Received 5 October 2018; published 1 April 2019)

\begin{abstract}
An unambiguous crystallographic structure solution for the observed phases II-VI of high pressure hydrogen does not exist due to the failure of standard structural probes at extreme pressure. In this work we propose that nuclear magnetic resonance spectroscopy provides a complementary structural probe for high pressure hydrogen. We show that the best structural models available for phases II, III, and IV of high pressure hydrogen exhibit markedly distinct nuclear magnetic resonance spectra which could therefore be used to discriminate amongst them. As an example, we demonstrate how nuclear magnetic resonance spectroscopy could be used to establish whether phase III exhibits polymorphism. Our calculations also reveal a strong renormalization of the nuclear magnetic resonance response in hydrogen arising from quantum fluctuations, as well as a strong isotope effect. As the experimental techniques develop, nuclear magnetic resonance spectroscopy can be expected to become a useful complementary structural probe in high pressure experiments.
\end{abstract}

DOI: $10.1103 /$ PhysRevLett.122.135501

Hydrogen has a central place in high pressure research [1] due to its abundance in astrophysical bodies [2] and its potential to exhibit exotic properties such as phononmediated high temperature superconductivity [3]. However, even the basic question of what are the crystal structures of the observed high pressure hydrogen phases remains elusive, largely due to the challenges faced by standard structure determination techniques under the extreme pressures reached in diamond anvil cells.

The lack of core electrons in hydrogen implies that $\mathrm{x}$ rays scatter from electrons located in molecular orbitals and cannot provide information about the individual positions of protons. Nonetheless, it has been possible to perform X-ray experiments up to pressures of $190 \mathrm{GPa}$ to establish that the hydrogen molecules are arranged in a hexagonal closed packed lattice up to those pressures $[4,5]$.

Protons and deuterons have significant neutron scattering cross sections, which might suggest that neutron diffraction would be an ideal probe for structural studies of high pressure hydrogen. However, the weakness of available neutron sources combined with the small samples in diamond anvil cells place practical limits to the applicability of this technique [6], and the highest pressure experiments reported in the hydrogen-deuterium system only reach $38 \mathrm{GPa}$ [7].

The most widely used structural probes of high pressure hydrogen are infrared (IR) and Raman spectroscopies.
Although these techniques have been extremely successful at identifying structural phase transitions in high pressure hydrogen [8-11], they only partially probe the lattice dynamics of phonons with vanishing wave vectors, and as a consequence are insufficient to determine the crystal structures of the underlying phases.

The difficulties faced by standard structure determination techniques in the case of high pressure hydrogen suggest that alternative methods could provide valuable complementary information. Nuclear magnetic resonance (NMR) spectroscopy probes the electronic response to applied magnetic fields, e.g., via the chemical shielding tensor $\boldsymbol{\sigma}$ in insulators, which relates the induced magnetic field $\mathbf{B}_{\text {in }}=-\boldsymbol{\sigma} \mathbf{B}_{0}$ at an atomic site to an applied external field $\mathbf{B}_{0}$. The chemical shielding tensor strongly depends on the local electronic configuration, and as a consequence encodes information about the local structure. This has led to the growing field of NMR crystallography, in which modeling and experiment are combined to solve crystal structures [12].

There has been long-term interest in combining NMR spectroscopy with diamond anvil cell high pressure experiments, and reports include studies of ortho-para hydrogen conversion up to $12.8 \mathrm{GPa}$ [13] and proton diffusion up to $6.8 \mathrm{GPa}$ [14]. Experiments are challenging due to a variety of factors, including weak signals, low resonator sensitivities, and poor access to the samples in the anvil cells, 
which have traditionally limited high pressure NMR spectroscopy experiments to a maximum of a few tens of GPa [15]. This situation has recently changed with the ground-breaking developments of Meier and co-workers, who exploiting magnetic flux tailoring Lenz lenses to amplify the magnetic field at the sample have measured hydrogen NMR spectra of paraffin up to $72 \mathrm{GPa}$ [16] and of $\mathrm{FeH}$ up to $200 \mathrm{GPa}$ [17]. The state of the art of high pressure NMR spectroscopy has recently been reviewed in Refs. [15,18]. These recent developments pave the path towards NMR spectroscopy experiments in the pressure range relevant for high pressure hydrogen phases II-VI. Phase II of hydrogen first appears at pressures in the range 73-110 GPa [19-21], and that of deuterium at even lower pressures of about $25 \mathrm{GPa}$ [22]. Phase III appears in the pressure range 150-170 GPa [23], and phase IV in the pressure range 200-220 GPa [24]. These are all within reach of the latest high pressure NMR spectroscopy experiments.

In this work we study the potential of NMR spectroscopy to probe the structure of high pressure hydrogen phases. In particular, we show that NMR chemical shieldings are markedly distinct between the various theoretical structural models proposed for hydrogen insulating phases II, III, and IV, and could therefore be used to assign the appropriate structure to these phases. We also expect that NMR spectroscopy could play a major role in other high pressure systems, including the hydrides which have recently been shown to exhibit some of the exotic properties initially proposed for pure hydrogen [25-27], and also compounds containing heavier elements that are present in the interiors of gas and rocky planets.

We consider six hydrogen structures. The $P 2_{1} c$ [28] and $P_{3} m$ [28] structures are candidates for phase II, which exists at low temperatures and at pressures up to around $150 \mathrm{GPa}$. The $C 2 / c$ [28] and $P 6_{1} 22$ [29] structures are candidates for phase III, which exists in a wide pressure range above about $150 \mathrm{GPa}$. The $P c$ [30] and $P c a 2_{1}$ [31] structures are candidates for phases IV and V, which appear around room temperature and at pressures higher than $220 \mathrm{GPa}$. All these structures have low enthalpies and Gibbs free energies [32-34], rendering them realistic candidates for the experimentally observed phases. Furthermore, several of their structural [29] and spectroscopic [35-38] features are consistent with corresponding experimental observations, although there are some outstanding discrepancies, particularly regarding the nature of their band gaps [39-41]. Available experimental and theoretical data are insufficient to unambiguously identify the correct structure associated with the observed experimental phases: for example both $C 2 / c$ and $P 6_{1} 22$ structures exhibit IR and Raman spectra consistent with phase III, and the $P c$ and $P c a 2_{1}$ structures consistent with phase IV, while their relative enthalpies are very similar.
To explore the possibility that NMR spectroscopy might provide complementary structural information, we have performed first principles density functional theory (DFT) calculations using the CASTEP package [42]. We have optimized the volumes and internal coordinates of the six structures by minimizing their enthalpies at pressures in the range 150-250 GPa, and using five different approaches, namely, the local density approximation (LDA) [43,44], the Perdew-Burke-Ernzerhof (PBE) approximation [45], the Becke-Lee-Yang-Parr (BLYP) approximation [46], the PBE approximation with the Tkatchenko-Scheffler van der Waals correction (PBE + TS) [47], and the hybrid Heyd-ScuseriaErnzerhof (HSE) approximation [48,49], to describe the exchange-correlation functional. We have then calculated the chemical shielding tensor using the gauge-including projector augmented waves (GIPAW) theory [50-52] as implemented in CASTEP.

The main result of our work is presented in Fig. 1, showing the isotropic chemical shieldings $\frac{1}{3} \operatorname{Tr}(\boldsymbol{\sigma})$ of the six considered structures calculated using the PBE functional. We also show the corresponding maximum and minimum absolute shielding anisotropies $\sigma_{\mathrm{SA}}=\left|\sigma_{33}-\frac{1}{2}\left(\sigma_{11}+\sigma_{22}\right)\right|$ in Table I. Isotropic line shapes could be obtained experimentally using magic angle spinning (MAS) [53], but we note that the precise parameters measured depend on the type of experiment performed, and we also provide magres files containing the full chemical shielding tensors with the Supplemental Material [54] to serve as the basis for further processing for direct comparison with future experiments.

Comparing the NMR spectra of the phase II candidate structures at $150 \mathrm{GPa}$ in Fig. 1, we observe that the $P 2_{1} c$ structure exhibits two closely spaced peaks centered around

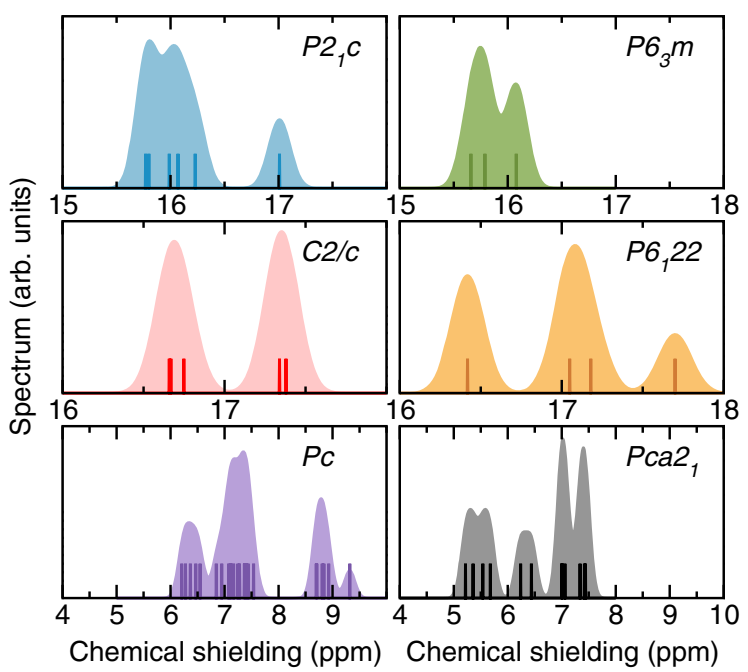

FIG. 1. Isotropic chemical shieldings calculated using the PBE functional at $150 \mathrm{GPa}$ for the $P 2_{1} c, P 6_{3} m, C 2 / c$, and $P 6_{1} 22$ structures, and at $250 \mathrm{GPa}$ for the $P c$ and $P c a 2_{1}$ structures. The vertical lines show the precise location of the calculated shieldings, while the wider spectra are the result of a convolution with a Gaussian function of $0.1 \mathrm{ppm}$ width. 
TABLE I. Shielding anisotropies calculated using the PBE functional at $150 \mathrm{GPa}$ for the $P 2_{1} c, P 6_{3} m, C 2 / c$, and $P 6_{1} 22$ structures, and at $250 \mathrm{GPa}$ for the $P c$ and $P c a 2_{1}$ structures.

\begin{tabular}{lcc}
\hline \hline & \multicolumn{2}{c}{ Shielding anisotropy $(\mathrm{ppm})$} \\
\cline { 2 - 3 } Structure & Maximum value & Minimum value \\
\hline$P 2_{1} c$ & 7.78 & 3.87 \\
$P 6_{3} m$ & 9.22 & 4.05 \\
$C 2 / c$ & 4.50 & 1.86 \\
$P 6_{1} 22$ & 4.49 & 1.50 \\
$P c$ & 6.56 & 2.93 \\
$P c a 2_{1}$ & 6.18 & 0.56 \\
\hline \hline
\end{tabular}

$16 \mathrm{ppm}$, and a separate narrower peak at around $17 \mathrm{ppm}$. There are six distinct atomic sites (each with four atoms) in the $P 2_{1} c$ structure, but five of these give similar chemical shieldings and result in the broad double peak, while the sixth site gives the lone peak at $17 \mathrm{ppm}$. By contrast, the $P 6_{3} m$ structure only exhibits two closely spaced peaks centered at $16 \mathrm{ppm}$, which are formed by three distinct chemical shieldings arising from the three independent atomic sites (two of these with six atoms each, and one with four atoms) in this structure. Analogous calculations using the LDA and BLYP functionals (see Supplemental Material [54]) consistently show a significantly broader spectrum for the $P 2_{1} c$ structure compared with the $P 6_{3} m$ structure. The different range and number of peaks between the two structures indicate that NMR spectroscopy could provide a promising tool to solve the structure of hydrogen phase II. It could also shed light on the potential polymorphism in phase II of deuterium [21,56].

Comparing the NMR spectra of the phase III candidate structures at $150 \mathrm{GPa}$ in Fig. 1, we observe that the $C 2 / \mathrm{c}$ structure exhibits two peaks centered at $16.70 \mathrm{ppm}$ and $17.35 \mathrm{ppm}$, which is a markedly different spectrum to that of the $P 6_{1} 22$ structure with three peaks at $16.40,17.10$, and $17.70 \mathrm{ppm}$. There are six distinct atomic sites (each with four atoms) in the $C 2 / c$ structure, which lead to six distinct chemical shieldings, but these cluster into two groups of three yielding the two observed peaks at the $0.1 \mathrm{ppm}$ resolution. There are also six distinct sites (each with six atoms) in the $P 6_{1} 22$ structure, yielding four distinct chemical shieldings (two pairs of sites yield the same shielding), two of which are similar and lead to the central peak, and the other two lead to peaks at the highest and lowest shieldings. We note that the peak at the lowest shielding has greater intensity than that at the highest shielding because the former arises from 12 atomic sites, while the latter from six. This clear difference between the NMR spectra of the $C 2 / c$ and $P 6_{1} 22$ structures could prove critical in understanding the properties of phase III. Theoretically, the $P 6_{1} 22$ structure has lower Gibbs free energy at temperatures in the range $0-300 \mathrm{~K}$ and at pressures below $200 \mathrm{GPa}$ and the $C 2 / c$ structure above that pressure, suggesting the possibility of polymorphism in phase III [29]. However, available experimental data are insufficient to confirm or reject this hypothesis, because both structures exhibit essentially indistinguishable IR and Raman spectra, consistent with experiment, and x-ray diffraction data are only available below $200 \mathrm{GPa}[4,5]$, suggesting a hexagonal structure consistent with $P 6_{1} 22$. The distinct NMR spectra exhibited by these two structures could unambiguously resolve the question of polymorphism in phase III. We note that the two-peak spectrum of the $C 2 / c$ structure and the three-peak spectrum of the $P 6_{1} 22$ structure is maintained if the calculations are performed using the LDA, PBE + TS, or HSE functionals, while the peaks move closer together when using the BLYP functional, but the latter describes the hydrogen bond more poorly (see Supplemental Material [54]).

Comparing the NMR spectra of the phase IV and V candidate structures at $250 \mathrm{GPa}$ in Fig. 1, we observe that the $P c$ structure exhibits a broad double peak between 6.00 and $7.75 \mathrm{ppm}$ and a narrower double peak between 8.50 and $9.50 \mathrm{ppm}$. There are 24 distinct atomic sites (each with two atoms) in the $P c$ structure, and each leads to a different value for the chemical shielding which results in the broad peaks observed in Fig. 1. The NMR spectra of the $P c a 2_{1}$ structure exhibits a continuum of peaks spanning the range from 5.00 to $7.75 \mathrm{ppm}$. These peaks arise from 12 distinct atomic sites (each with four atoms), a smaller number compared to the $P c$ structure. The only available experimental information on phases IV and V is based on their Raman and IR spectra [8,9], which are largely consistent with those of both $P c$ and $P c a 2_{1}$ structures [31,35]. Again, our results show that NMR spectroscopy could provide further evidence to disentangle the underlying structure of these phases of hydrogen. We note that using the LDA functional leads to a similar picture to that shown in Fig. 1, in which the $P c$ NMR spectrum spans a wider range of chemical shieldings than the spectrum of the $P c a 2_{1}$ structure. The comparison using the BLYP functional is not possible because the $P c a 2_{1}$ structure exhibits metallic behavior in that case (see Supplemental Material [54]).

We have repeated the chemical shielding tensor calculations of the phase III candidate $C 2 / c$ and $P 6_{1} 22$ structures as a function of pressure in the range 150 to $250 \mathrm{GPa}$, and the results are depicted in Fig. 2. There is a relatively strong shift of the NMR peak positions as a function of pressure from around $17 \mathrm{ppm}$ at $150 \mathrm{GPa}$ to about $3 \mathrm{ppm}$ at $250 \mathrm{GPa}$. Nonetheless, the relative shieldings of the spectra depicted in Fig. 1 for $150 \mathrm{GPa}$ are maintained at all the considered pressures, suggesting that experiments at any pressure in which phase III is observed should be sufficient to fully explore the relation between the NMR spectra and the underlying structures. We also remark that NMR spectroscopy experiments do not measure absolute shieldings, but instead relative shifts with respect to some reference structure, and therefore the 


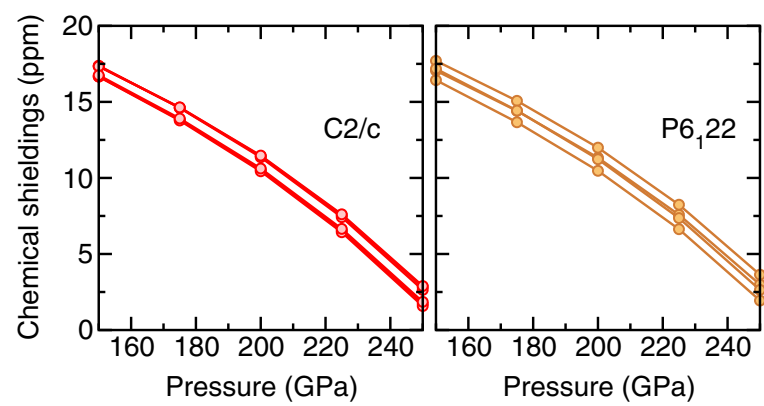

FIG. 2. Isotropic chemical shieldings of the $C 2 / c$ and $P 6_{1} 22$ structures calculated using the PBE functional as a function of pressure.

relevant quantity in our predictions is the relative position of the peaks, not their absolute value.

All calculations reported so far have been performed at the static lattice level of theory. However, hydrogen is the lightest atom and quantum zero point motion is known to strongly renormalize its energetic [33], structural [57], optical [39,41], and vibrational [35,37] properties. We have therefore performed chemical shielding tensor calculations for the $C 2 / c$ and $P 6_{1} 22$ structures including the effects of quantum zero-point motion. The initial step is the calculation of the lattice dynamics at the harmonic level of theory, which we have performed using DFT as implemented in the CASTEP package, and using the finite displacement method in conjunction with nondiagonal supercells [58]. Anharmonic lattice dynamics contributions are known to be very important for calculating the relative energy of different hydrogen structures, but this is mostly due to the small energy differences between structures rather than a significant anharmonic energy, which is only about $5 \%$ of the harmonic energy [33]. We have therefore neglected anharmonic terms in our lattice dynamics calculations.

We then calculate the zero point quantum renormalization of the chemical shielding tensor using the stochastic approach described in Refs. [59-61], and accelerating the sampling by exploiting thermal lines $[62,63]$. The isotropic chemical shieldings of the $C 2 / c$ and $P 6_{1} 22$ structures calculated with the inclusion of quantum zero-point motion are depicted in Fig. 3. Zero-point motion leads to a strong renormalization of the peak positions of about $7 \mathrm{ppm}$, confirming that quantum fluctuations are strong in hydrogen. However, the number and relative position of the peaks in the spectra of the two structures remain similar to that of the static lattice results. This suggests that, although quantum zero point motion strongly renormalizes the electronic response to magnetic fields in hydrogen, the results depicted in Fig. 1 showing the differences in the NMR spectra between different high pressure hydrogen candidate structures remain valid.

Figure 3 also shows the quantum zero-point renormalization of the chemical shielding corresponding to the

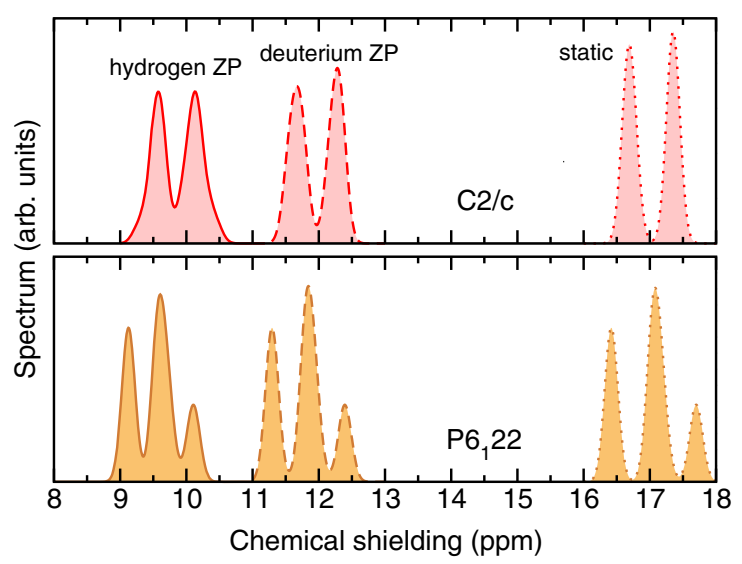

FIG. 3. Isotropic chemical shieldings of $C 2 / c$ and $P 6_{1} 22$ calculated using the PBE functional at $150 \mathrm{GPa}$. The dotted line distributions show the static lattice results, and the dashed and solid line distributions show the results including quantum zero-point (ZP) motion for deuterium and hydrogen, respectively. The results have been convoluted with a Gaussian function of $0.1 \mathrm{ppm}$ width.

deuterium isotope. As a consequence of the large mass difference between hydrogen and deuterium, the zerotemperature chemical shieldings exhibit a strong isotope effect. This suggests that NMR spectroscopy could also be used to explore the isotopic composition of high pressure hydrogen phases. In addition, we note that the deuterium nucleus is quadrupolar and it will therefore be subject to an electric field gradient (relevant parameters listed in the Supplemental Material [54]). This implies that in experiments without MAS, the spectral lines will be broadened by the quadrupolar interaction in deuterium.

Our results indicate that NMR spectroscopy is a promising technique for elucidating the structures of the observed phases of high pressure hydrogen. Direct observation of the calculated isotropic chemical shieldings would require MAS of a proton sample in a diamond anvil cell. Although MAS has been successfully combined with diamond anvil cells, the large rotor speeds needed for proton signals might pose technical challenges due to the small size rotors required. Alternatively, MAS experiments on deuterium samples with residual protons would allow slower spinning and thus larger rotors, simplifying experiments. We note that good sensitivities are obtained in samples with proton concentrations as low as 1\% [64]. Finally, the technically simpler experiments would be static, but in those the presence of anisotropies implies that measurements should exploit differences in proton chemical shielding anisotropies or deuterium quadrupolar couplings rather than isotropic chemical shieldings. Magres files containing the full chemical shielding tensors are published with the Supplemental Material [54].

In conclusion, we propose that nuclear magnetic resonance could become a valuable probe to identify the underlying structures of the observed high pressure phases 
of solid hydrogen. We have shown that isotropic NMR spectra would be particularly useful as the chemical shieldings are markedly different between the different structural models available for hydrogen phases II, III, and IV and V. For example, they could be used to resolve questions such as the potential polymorphism of phase III or the precise structural sequence in phases IV and V. Our results pave the path for the use of NMR spectroscopy in structure determination in other high pressure systems, both for hydrogen-rich compounds or for other compounds containing heavier atoms.

B. M. acknowledges support from the Winton Programme for the Physics of Sustainability, and from Robinson College, Cambridge, and the Cambridge Philosophical Society for a Henslow Research Fellowship. S.E. A. and C. J. P. are supported by the Royal Society through a Royal Society Wolfson Research Merit award. Part of the calculations were performed using the Archer facility of the UK's national high-performance computing service (for which access was obtained via the UKCP consortium [EP/P022596/1]).

*bm418@cam.ac.uk

[1] J. M. McMahon, M. A. Morales, C. Pierleoni, and D. M. Ceperley, The properties of hydrogen and helium under extreme conditions, Rev. Mod. Phys. 84, 1607 (2012).

[2] N. Nettelmann, A. Becker, B. Holst, and R. Redmer, Jupiter models with improved $a b$ initio hydrogen equation of state (H-REOS.2), Astrophys. J. 750, 52 (2012).

[3] N. W. Ashcroft, Metallic hydrogen: A High-Temperature Superconductor? Phys. Rev. Lett. 21, 1748 (1968).

[4] Y. Akahama, M. Nishimura, H. Kawamura, N. Hirao, Y. Ohishi, and K. Takemura, Evidence from x-ray diffraction of orientational ordering in phase III of solid hydrogen at pressures up to $183 \mathrm{GPa}$, Phys. Rev. B 82, 060101 (2010).

[5] Y. Akahama, Y. Mizuki, S. Nakano, N. Hirao, and Y. Ohishi, Raman scattering and $\mathrm{x}$-ray diffraction studies on phase III of solid hydrogen, J. Phys. Conf. Ser. 950, 042060 (2017).

[6] M. Guthrie, Future directions in high-pressure neutron diffraction, J. Phys. Condens. Matter 27, 153201 (2015).

[7] I. Goncharenko and P. Loubeyre, Neutron and x-ray diffraction study of the broken symmetry phase transition in solid deuterium, Nature (London) 435, 1206 (2005).

[8] M. I. Eremets and I. A. Troyan, Conductive dense hydrogen, Nat. Mater. 10, 927 (2011).

[9] P. Dalladay-Simpson, R. T. Howie, and E. Gregoryanz, Evidence for a new phase of dense hydrogen above 325 gigapascals, Nature (London) 529, 63 (2016).

[10] R. P. Dias, O. Noked, and I. F. Silvera, New Phases and Dissociation-Recombination of Hydrogen Deuteride to 3.4 Mbar, Phys. Rev. Lett. 116, 145501 (2016).

[11] R. P. Dias and I. F. Silvera, Observation of the WignerHuntington transition to metallic hydrogen, Science 355, 715 (2017).
[12] E. Salager, G. M. Day, R. S. Stein, C. J. Pickard, B. Elena, and L. Emsley, Powder crystallography by combined crystal structure prediction and high-resolution ${ }^{1} \mathrm{H}$ solidstate NMR spectroscopy, J. Am. Chem. Soc. 132, 2564 (2010).

[13] M. G. Pravica and I. F. Silvera, NMR Study of Ortho-Para Conversion at High Pressure in Hydrogen, Phys. Rev. Lett. 81, 4180 (1998).

[14] S.-H. Lee, M. S. Conradi, and R. E. Norberg, Molecular motion in solid $\mathrm{H}_{2}$ at high pressures, Phys. Rev. B 40, 12492 (1989).

[15] T. Meier, At its Extremes: NMR at Giga-Pascal Pressures (Academic Press, London, 2018), Chap. 1, pp. 1-74.

[16] T. Meier, N. Wang, D. Mager, J. G. Korvink, S. Petitgirard, and L. Dubrovinsky, Magnetic flux tailoring through Lenz lenses for ultrasmall samples: A new pathway to highpressure nuclear magnetic resonance, Sci. Adv. 3, eaao5242 (2017).

[17] T. Meier, F. Trybel, S. Khandarkhaeva, G. SteinleNeumann, S. Chariton, T. Fedotenko, S. Petitgirard, M. Hanfland, K. Glazyrin, N. Dubrovinskaia, and L. Dubrovinsky, Pressure induced hydrogen-hydrogen interaction in metallic $\mathrm{FeH}$ revealed by NMR, arXiv: 1902.03182.

[18] T. Meier, Journey to the centre of the Earth: Jules Vernes dream in the laboratory from an NMR perspective, Prog. Nucl. Magn. Reson. Spectrosc. 106-107, 26 (2018).

[19] H. E. Lorenzana, I. F. Silvera, and K. A. Goettel, Orientational Phase Transitions in Hydrogen at Megabar Pressures, Phys. Rev. Lett. 64, 1939 (1990).

[20] M. Hanfland, R. J. Hemley, and H.-k. Mao, Novel Infrared Vibron Absorption in Solid Hydrogen at Megabar Pressures, Phys. Rev. Lett. 70, 3760 (1993).

[21] X.-D. Liu, R. T. Howie, H.-C. Zhang, X.-J. Chen, and E. Gregoryanz, High-Pressure Behavior of Hydrogen and Deuterium at Low Temperatures, Phys. Rev. Lett. 119, 065301 (2017).

[22] I. F. Silvera and R. J. Wijngaarden, New Low-Temperature Phase of Molecular Deuterium at Ultrahigh Pressure, Phys. Rev. Lett. 47, 39 (1981).

[23] R. J. Hemley and H. K. Mao, Phase Transition in Solid Molecular Hydrogen at Ultrahigh Pressures, Phys. Rev. Lett. 61, 857 (1988).

[24] R. T. Howie, C. L. Guillaume, T. Scheler, A. F. Goncharov, and E. Gregoryanz, Mixed Molecular and Atomic Phase of Dense Hydrogen, Phys. Rev. Lett. 108, 125501 (2012).

[25] N. W. Ashcroft, Hydrogen Dominant Metallic Alloys: High Temperature Superconductors, Phys. Rev. Lett. 92, 187002 (2004).

[26] D. Duan, Y. Liu, F. Tian, D. Li, X. Huang, Z. Zhao, H. Yu, B. Liu, W. Tian, and T. Cui, Pressure-induced metallization of dense $\left(\mathrm{H}_{2} \mathrm{~S}\right)_{2} \mathrm{H}_{2}$ with high- $\mathrm{T}_{\mathrm{c}}$ superconductivity, Sci. Rep. 4, 6968 (2014).

[27] A. P. Drozdov, M. I. Eremets, I. A. Troyan, V. Ksenofontov, and S. I. Shylin, Conventional superconductivity at 203 kelvin at high pressures in the sulfur hydride system, Nature (London) 525, 73 (2015).

[28] C. J. Pickard and R. J. Needs, Structure of phase III of solid hydrogen, Nat. Phys. 3, 473 (2007).

[29] B. Monserrat, R. J. Needs, E. Gregoryanz, and C. J. Pickard, Hexagonal structure of phase III of solid hydrogen, Phys. Rev. B 94, 134101 (2016). 
[30] C. J. Pickard, M. Martinez-Canales, and R. J. Needs, Density functional theory study of phase IV of solid hydrogen, Phys. Rev. B 85, 214114 (2012).

[31] B. Monserrat, N. D. Drummond, P. Dalladay-Simpson, R. T. Howie, P. López Ríos, E. Gregoryanz, C. J. Pickard, and R. J. Needs, Structure and Metallicity of Phase V of Hydrogen, Phys. Rev. Lett. 120, 255701 (2018).

[32] S. Azadi, B. Monserrat, W. M. C. Foulkes, and R. J. Needs, Dissociation of High-Pressure Solid Molecular Hydrogen: A Quantum Monte Carlo and Anharmonic Vibrational Study, Phys. Rev. Lett. 112, 165501 (2014).

[33] N. D. Drummond, B. Monserrat, J. H. Lloyd-Williams, P. López Ríos, C. J. Pickard, and R. J. Needs, Quantum Monte Carlo study of the phase diagram of solid molecular hydrogen at extreme pressures, Nat. Commun. 6, 7794 (2015).

[34] J. McMinis, R. C. Clay, D. Lee, and M. A. Morales, Molecular to Atomic Phase Transition in Hydrogen under High Pressure, Phys. Rev. Lett. 114, 105305 (2015).

[35] I. B. Magdáu and G. J. Ackland, Identification of highpressure phases III and IV in hydrogen: Simulating Raman spectra using molecular dynamics, Phys. Rev. B 87, 174110 (2013).

[36] R. T. Howie, I. B. Magdáu, A. F. Goncharov, G. J. Ackland, and E. Gregoryanz, Phonon Localization by Mass Disorder in Dense Hydrogen-Deuterium Binary Alloy, Phys. Rev. Lett. 113, 175501 (2014).

[37] R. Singh, S. Azadi, and T. D. Kühne, Anharmonicity and finite-temperature effects on the structure, stability, and vibrational spectrum of phase III of solid molecular hydrogen, Phys. Rev. B 90, 014110 (2014).

[38] I. B. Magdáu and G. J. Ackland, Infrared Peak Splitting from Phonon Localization in Solid Hydrogen, Phys. Rev. Lett. 118, 145701 (2017).

[39] M. A. Morales, J. M. McMahon, C. Pierleoni, and D. M. Ceperley, Towards a predictive first-principles description of solid molecular hydrogen with density functional theory, Phys. Rev. B 87, 184107 (2013).

[40] S. Azadi, N. D. Drummond, and W. M. C. Foulkes, Nature of the metallization transition in solid hydrogen, Phys. Rev. B 95, 035142 (2017).

[41] S. Azadi, R. Singh, and T. D. Kühne, Nuclear quantum effects induce metallization of dense solid molecular hydrogen, J. Comput. Chem. 39, 262 (2018).

[42] S. J. Clark, M. D. Segall, C. J. Pickard, P. J. Hasnip, M. I. J. Probert, K. Refson, and M.C. Payne, First principles methods using CASTEP, Z. Kristallogr. 220, 567 (2005).

[43] D. M. Ceperley and B. J. Alder, Ground State of the Electron Gas by a Stochastic Method, Phys. Rev. Lett. 45, 566 (1980).

[44] J. P. Perdew and Y. Wang, Accurate and simple analytic representation of the electron-gas correlation energy, Phys. Rev. B 45, 13244 (1992).

[45] J. P. Perdew, K. Burke, and M. Ernzerhof, Generalized Gradient Approximation made Simple, Phys. Rev. Lett. 77, 3865 (1996).

[46] A. D. Becke, Density-functional exchange-energy approximation with correct asymptotic behavior, Phys. Rev. A 38, 3098 (1988).

[47] A. Tkatchenko and M. Scheffler, Accurate Molecular van der Waals Interactions from Ground-State Electron Density and Free-Atom Reference Data, Phys. Rev. Lett. 102, 073005 (2009).

[48] J. Heyd, G. E. Scuseria, and M. Ernzerhof, Hybrid functionals based on a screened Coulomb potential, J. Chem. Phys. 118, 8207 (2003).

[49] J. Paier, M. Marsman, K. Hummer, G. Kresse, I. C. Gerber, and J.G. Ángyán, Screened hybrid density functionals applied to solids, J. Chem. Phys. 124, 154709 (2006).

[50] C. J. Pickard and F. Mauri, All-electron magnetic response with pseudopotentials: NMR chemical shifts, Phys. Rev. B 63, 245101 (2001).

[51] J. R. Yates, C. J. Pickard, and F. Mauri, Calculation of NMR chemical shifts for extended systems using ultrasoft pseudopotentials, Phys. Rev. B 76, 024401 (2007).

[52] C. Bonhomme, C. Gervais, F. Babonneau, C. Coelho, F. Pourpoint, T. Azaïs, S. E. Ashbrook, J. M. Griffin, J. R. Yates, F. Mauri, and C. J. Pickard, First-principles calculation of NMR parameters using the gauge including projector augmented wave method: A chemists point of view, Chem. Rev. 112, 5733 (2012).

[53] E. R. Andrew, A. Bradbury, and R. G. Eades, Nuclear magnetic resonance spectra from a crystal rotated at high speed, Nature (London) 182, 1659 (1958).

[54] See Supplemental Material at http://link.aps.org/ supplemental/10.1103/PhysRevLett.122.135501 for computational details and MAGRES files, which includes Ref. [56].

[55] W. D. Knight, Nuclear magnetic resonance shift in metals, Phys. Rev. 76, 1259 (1949).

[56] A. F. Goncharov, J. H. Eggert, I. I. Mazin, R. J. Hemley, and H.-k. Mao, Raman excitations and orientational ordering in deuterium at high pressure, Phys. Rev. B 54, R15590 (1996).

[57] G. Rillo, M. A. Morales, D. M. Ceperley, and C. Pierleoni, Coupled electron-ion Monte Carlo simulation of hydrogen molecular crystals, J. Chem. Phys. 148, 102314 (2018).

[58] J. H. Lloyd-Williams and B. Monserrat, Lattice dynamics and electron-phonon coupling calculations using nondiagonal supercells, Phys. Rev. B 92, 184301 (2015).

[59] S. Rossano, F. Mauri, C. J. Pickard, and I. Farnan, Firstprinciples calculation of ${ }^{17} \mathrm{O}$ and ${ }^{25} \mathrm{Mg}$ NMR shieldings in $\mathrm{MgO}$ at finite temperature: Rovibrational effect in solids, J. Phys. Chem. B 109, 7245 (2005).

[60] B. Monserrat, R. J. Needs, and C. J. Pickard, Temperature effects in first-principles solid state calculations of the chemical shielding tensor made simple, J. Chem. Phys. 141, 134113 (2014).

[61] R. Nemausat, D. Cabaret, C. Gervais, C. Brouder, N. Trcera, A. Bordage, I. Errea, and F. Mauri, Phonon effects on X-ray absorption and nuclear magnetic resonance spectroscopies, Phys. Rev. B 92, 144310 (2015).

[62] B. Monserrat, Vibrational averages along thermal lines, Phys. Rev. B 93, 014302 (2016).

[63] B. Monserrat, Electron-phonon coupling from finite differences, J. Phys. Condens. Matter 30, 083001 (2018).

[64] A. E. McDermott, F. J. Creuzet, A. C. Kolbert, and R. G. Griffin, High-resolution magic-angle-spinning NMR spectra of protons in deuterated solids, J. Magn. Reson. 98, 408 (1992). 